\title{
Towards multimodal BCIs: the impact of peripheral control on motor cortex activity and sense of agency
}

\author{
Tristan Venot ${ }^{1}$, Marie Constance Corsi ${ }^{2}$, Ludovic Saint-Bauzel ${ }^{3}$ and Fabrizio de Vico Fallani ${ }^{4}$
}

\begin{abstract}
In the recent years, brain computer interfaces (BCI) using motor imagery have shown some limitations regarding the quality of control. In an effort to improve this promising technology, some studies intended to develop hybrid BCI with other technologies such as eye tracking which shows more reliability. However, the use of an eye tracker in the control of a robot might affect by itself the sense of agency (SoA) and the brain activity in the regions used for motor imagery (MI). Here, we explore the link between the sense of agency and the activity of the motor cortex. For this purpose, we used of a virtual arm projected on a surface which is either controlled by motion capture or controlled by gaze using an eye tracker. We found out that there is an activity in the motor cortex during the task of control by gaze and that having control over a projected robotic arm presents significant differences with the situation of observing the robot moving.
\end{abstract}

\section{INTRODUCTION}

Brain Computer Interface is a wide field of study [1] that focuses on extracting brain signals and the ways to interconnect them with a computer. Despite the breakthrough in machine learning and optimisation techniques [2], it remains extremely difficult to find robust control over a robot for instance in the case of BCI based on scalp EEG using motor imagery (the mental process of imaging a movement without actually performing it [3]). To strengthen the BCI, few studies tried to combine it with other more reliable technologies such as eye tracker which shows less variability across subjects [4] [5]. Despite expected performance improvement, the generation of overlapping brain activity processes is still poorly understood.

Indeed, the literature is unclear whether the control by gaze of the robot activates the zone used for motor imagery task. Furthermore, this goes to the extent of any sentiment of control which could produce an activity in the motor cortex activity. Hence we need to address the possible link between the sense of agency, " a subjective experience of action control, intention, [...] " [6], and motor cortex activity.

To address this question, we created a protocol with 3 tasks, two types of control of a virtual robotic arm by movement or gaze and a robot observation task.

\footnotetext{
${ }^{1} \mathrm{~T}$. Venot, Inria, Aramis project-team, Paris Brain Institute - Institut du Cerveau (ICM), Inserm U 1127, CNRS UMR 7225, Sorbonne Université, F-75013, Paris, France (phone: +33 6-17-81-09-81;e-mail: tristan.venot@inria.fr).

${ }^{2}$ M.C. Corsi, Paris Brain Institute - Institut du Cerveau (ICM), Inserm U 1127, CNRS UMR 7225, Sorbonne Université, Inria, Aramis project-team, F-75013, Paris, France (e-mail: marieconstance.corsi@icm-institute.org

${ }^{3}$ L. Saint-Bauzel ISIR,CNRS UMR 7222, 75005 Paris France (e-mail: ludovic.saint-bauzel@sorbonne-universite.fr).

${ }^{4}$ F. de Vico Fallani, Inria, Aramis project-team, Paris Brain Institute Institut du Cerveau (ICM), Inserm U 1127, CNRS UMR 7225, Sorbonne Université, F-75013, Paris, France(e-mail: fabrizio.de-vico-fallani@ inria.fr).
}

\section{METHOD, MATERIAL AND PROTOCOL}

\section{A. Strategy employed}

Study's principal criterion is the level of agency. There are three level of control:

1) Mirror control

2) Control by gaze

3) No control

The level of agency is given by a questionnaire, a translation of the Wegner et al. (2004) [7], which provides a score for the different conditions. Our variable of interest is the significant differences of activation pattern between resting state, mirror control and control by gaze state in $\alpha$ and $\beta$ frequency bands $(8-30 \mathrm{~Hz})$ associated with motor action.

\section{B. Material}

We created a structure to support an eye tracking device and a projector to display a virtual robot. Through the frame, a virtual scene is projected composed of virtual moving red targets and a robotic arm. We use a Tobii Pro X3 in our experimentation. The second part of the user's environment is a Kinect v1 mounted on a tripod coupled with a screen where the same virtual environment is displayed. The EEG cap used is a Enobio 8 electrodes.

\section{Experimental protocol}

Eight healthy subjects (aged $26 \pm 3$ years, 4 men) volunteered for the experience. They all came from scientific background and were not familiar with eye tracking technologies. One of the subjects was not a french speaker therefore he received the original questionnaire. They all signed informed consent according to institutional guidelines. The protocol was reviewed by Sorbonne's ethical committee. The EEG cap is installed on the placement $\mathrm{Cz}, \mathrm{FC} 1, \mathrm{C} 1, \mathrm{CP} 1, \mathrm{P} 3, \mathrm{CP} 5$, $\mathrm{C} 3$ and FC5. EEG recordings were performed at a sampling rate of $500 \mathrm{~Hz}$ and a bandwidth of $0-60 \mathrm{~Hz}$. A robotic arm moving accordingly to the movement of the subjects' right arm was displayed on a monitor, virtual objects were moving randomly on the screen as targets to catch. During 4 sessions of 5 minutes, subjects were required either to stay still or to move their arm and observe the robotic arm moving. This will result in a separation between feeling and visualisation, it will be the feeling of the real arm but the visualisation of the robotic arm. They were then placed in front of a frame where they controlled the robotic arm with their gaze. The arm was projected on a tilted plane. The subjects were asked either to control the robot or to rest for 2 sessions of 5 minutes. We still have a SoA which is no more linked to 
motor control. Then, the subjects were presented the same robotic arm moving by itself. The subjects were asked either to control the robot or to rest for 2 sessions of 5 minutes. Between each set of tasks, the subjects answered a SoA questionnaire which assessed their sensation of control over the robotic arm. In total, the subjects performed the motor activity 36 times, the control by gaze 24 times, the absence of control 15 times and the resting state 45 times. This difference between the number of trials for each condition is due to time constraints and to avoid subjects' tiredness.

\section{Mirror control}

To perform control by motion capture, we used joints estimation (shoulder, elbow, wrist) by computing a distance transformation as presented by Quoc and al [8]. From this joints estimation we obtained the vectors chest to shoulder, shoulder to elbow and elbow to wrist. This vectors were reproduced by the robotic arm. We achieved direct control of the virtual arm by mimicking the movement of the user. We refer to this type of control as "Mirror Control" because the robotic arm acts as a reflection in a mirror. To find a common pattern in the movement of a grasping task, we performed a Principal Component Analysis on the vectors obtained before in order. Then, we extracted the implication of each joints in the movement that will be used later on to control the behaviour of the arm when it is controlled by the gaze.

\section{E. Control by gaze}

From the eye tracker, we obtained a $2 \mathrm{D}$ estimation of the gaze position on the surface. To obtain stable smooth movements that can integrate the characteristics of the human movement, we used a pseudo inverse of a damped Jacobian [9]. As a result the user focused his gaze for a period of at least $200 \mathrm{~ms}$ on a targeted area which could be a moving target and the effector (the last part of the arm) reached the desired position.

\section{F. No control}

To obtain a robot moving by itself, we randomized the value of joint angles to reach every time a different position. The subject could just observe the movement without being involved.

\section{G. Data Analysis}

The EEG data were processed to extract their spectral density dynamic, we used Matlab with the EEGlab[10] plugin for ICA treatment and Brainstorm [11] for statistical analysis. We computed the power spectrum using Welch method for each trial and in average for every conditions. To perform the analysis at the group level regarding the different conditions, we performed a comparison on the average of all the trials by conditions using the two tailed Wilcoxon test in $\alpha(8-12 \mathrm{~Hz})$ and $\beta(15-29 \mathrm{~Hz})$ bands. Secondly, we performed a permutation paired student t-test on the different conditions using a subset of samples to have the same number of trials in each condition for each subjects.

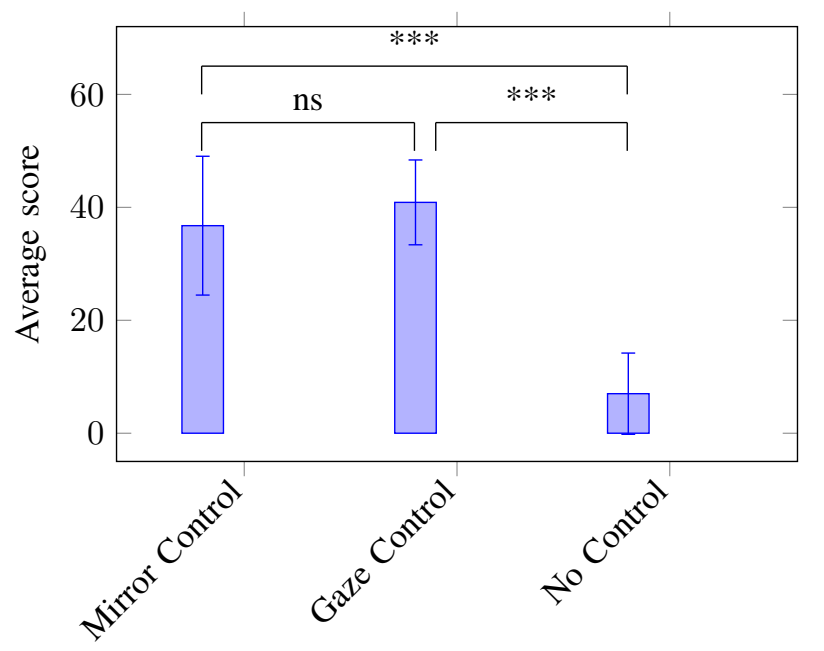

Fig. 1. Average score of the sense of agency for the different tasks between subjects, significant differences $(p<0.001)$ for Mirror Control vs No Control and Gaze Control vs No Control

\section{RESULTS}

We observed significant higher SoA for the two active conditions (Mirror Control and Gaze Control) as compared to No Control Fig 1 . There were no significant differences between conditions Mirror Control and Gaze control. Our comparison on spectral density between conditions on average across subjects allowed us to be aware of a trend. We established the certainty of the motor activity (Mirror control) compared to resting state resulting in a significant $(\mathrm{p}<0.05)$ decrease of power (negative z-value) in the $\alpha$ and $\beta$ bands. Secondly, we could already observe similar results for control by gaze compared to resting state with a significant decrease of power $(\mathrm{p}<0.05)$ in both frequency bands. However between the situation no control and resting state, we only observed significant decrease of power $(\mathrm{p}<0.05)$ in the $\alpha$ band.

We compared the spectral density subject by subject between the different conditions, we observed a significant difference of activity in the alpha and beta bands in the motor region $(\mathrm{p}<0.05)$ and negative t-values indicating a decrease of power. We established the number of times we observe negative t-values for each electrodes from a subject to another as shown in Fig 2 and evaluated the most interesting $\mathrm{t}$-values for each comparison Tab. If As expected, the decrease of power corresponding to negative $\mathrm{t}$-values occurred in most of the significant $(\mathrm{p}<0.05)$ case $(6$ subjects) between Mirror control and resting state. Between the condition control by gaze and resting state for each subjects, we observed a significant difference of spectral activity for 5 of the subjects $(\mathrm{p}<0.05)$ and negative $\mathrm{t}$-values indicating a decrease of power. This indicates that being in control of the robot with the gaze can have an impact on the motor cortex activity. Mirror control and control by gaze present similar results both on sense of agency rating and in number of occurrences.

Between no control and resting state, we observed for 4 of the subjects in the $\alpha$ band negative t-values $(\mathrm{p}<$ 

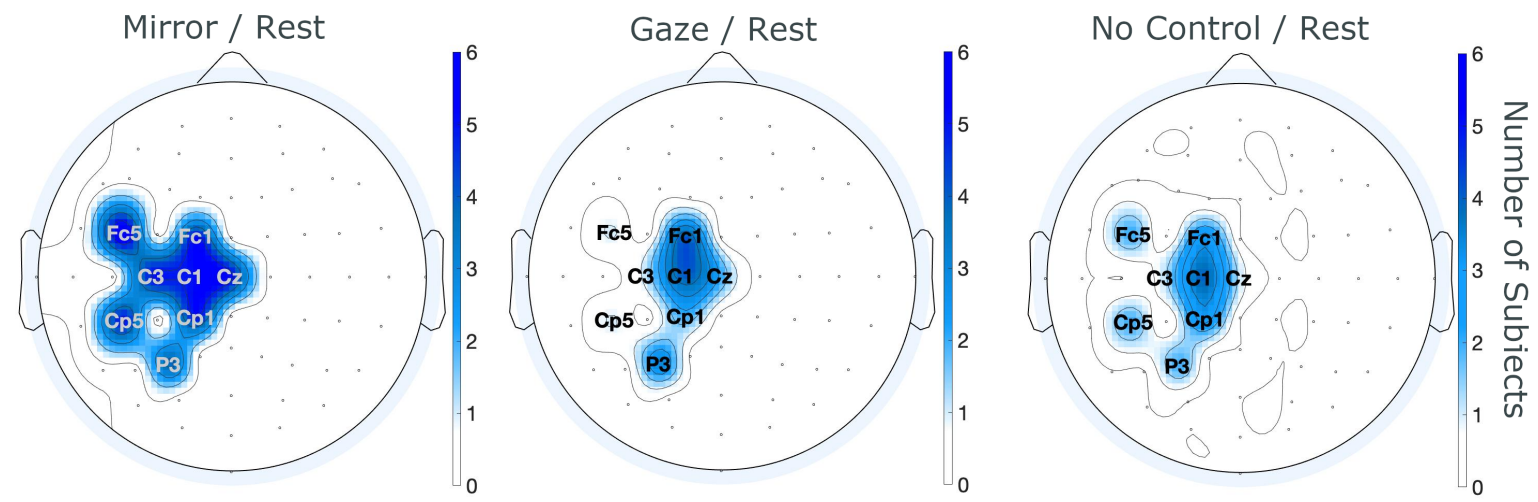

Fig. 2. Group-level EEG power significant differences between conditions. Coloured regions in the scalp-maps represent the number of subjects exhibiting negative t-values $(p<0.05$, FDR corrected) at each electrode position.

$0.05)$ indicating a possible activity in the motor cortex. By comparing conditions control by gaze and no control Fig 3 , we observed as well differences of activity $(p<0.05)$ for all the subjects and negative t-values for 6 subjects. Two of the subjects (Sub6,Sub8) presented positive t-values $(\mathrm{p}<$ $0.05)$ in the $\alpha$ band. The activation of the zone associated with movement planning (and motor imagery) could mean that in the performance of a mental task of control (without moving), similar areas that are typically used for motor imagery would be solicited, too.

\section{Discussion}

In hybrid MI BCI context, the link between the observation of a robot moving or its control by gaze and the activation of the motor cortex region in bands associated with attention and motor activity must be addressed. Here, we show significant differences of spectral density between resting state and the three other conditions (eg Mirror Control, Control by gaze and No control) with negative t-values. This indicates a decrease of power in the frequency bands of interest $(\alpha$ and $\beta$ ), a marker of motor activity. Furthermore, the notion of control established by the SoA scoring does not have to be necessary linked to the notion of motor action to generate an activity in the motor cortex. The difference between no-control and resting state can be explained by two different reasons. First, motor activity can be triggered by the observation of someone else's movement [12], a similar mechanism might occur when observing a robotic arm moving which was controlled before. Secondly, the no control phase comes right after the control by gaze phase. Due to the instruction, subjects are still trying to control the robotic arm at the beginning of the session resulting in an activity in the same region.

We can make the hypothesis that the no control state is closer to the resting state, which would explain why there is a decrease of power density in the $\alpha$ and $\beta$ bands (negative t-values) for 6 of the subjects Fig 3 . The two subjects presenting an increase in the $\alpha$ band (positive t-values) might have been paying more attention to the robot moving freely, this being an interpretation of the alpha band in accordance with the literature [13]. However we must keep in mind that we only have 8 electrodes over the sensorimotor area, and we cannot exclude other more distributed significant activation. We conclude that in the context of MI BCI mixed with eye tracker, there might be possible overlap of brain activity between motor imagery and control by gaze. It is important to remark that two subjects (Sub 4, Sub 5) were left-handed but the virtual arm was placed on the right side of the projected scene and its behaviour was based on right arm movements. This laterality could affect the performance of the subjects in their motor imagery task [14]. Taken together, our results suggest possible activation of the motor cortex due to direct or indirect sense of control. In future development, we shall investigate the best strategy to intertwine eye tracker and MI BCI for the control of a robot.

\section{ACKNOWLEDGEMENT}

The authors warmly thank PhD T. Cattai and J. Gonzalez Astudillo for their help and advice in the realisation of this paper.

\section{REFERENCES}

[1] Brain-Computer Interfaces: Principles and Practice. Oxford, New York: Oxford University Press, Mar. 1, 2012. 424 pp. ISBN: 978-0-19-538885-5.

[2] F. Lotte et al. "A review of classification algorithms for EEG-based brain-computer interfaces: a 10 year update". In: Journal of Neural Engineering 15.3 (2018).

[3] A. Guillot and C. Collet. "Contribution from neurophysiological and psychological methods to the study of motor imagery". In: Brain Research Reviews 50.2 (2005), pp. 387-397.

[4] Y. Wang et al. "A Human-Robot Interaction System Based on Hybrid Gaze Brain-Machine Interface and Shared Control". In: Jiqiren/Robot 40 (2018), pp. 431439.

[5] L. Schiatti et al. "Soft brain-machine interfaces for assistive robotics: A novel control approach". In: 2017 International Conference on Rehabilitation Robotics. IEEE, 2017, pp. 863-869. 

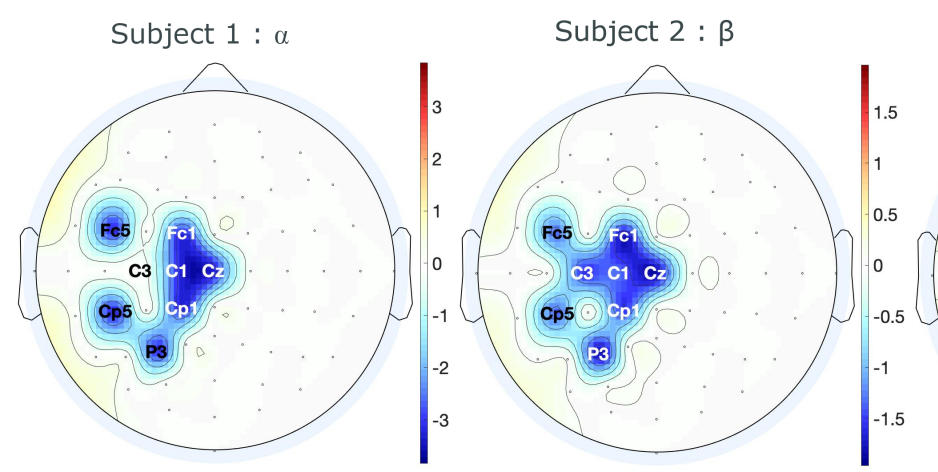

Subject $3: \alpha$

Subject $4: \beta$

Subject $5: \alpha$

Subject $6: \alpha$
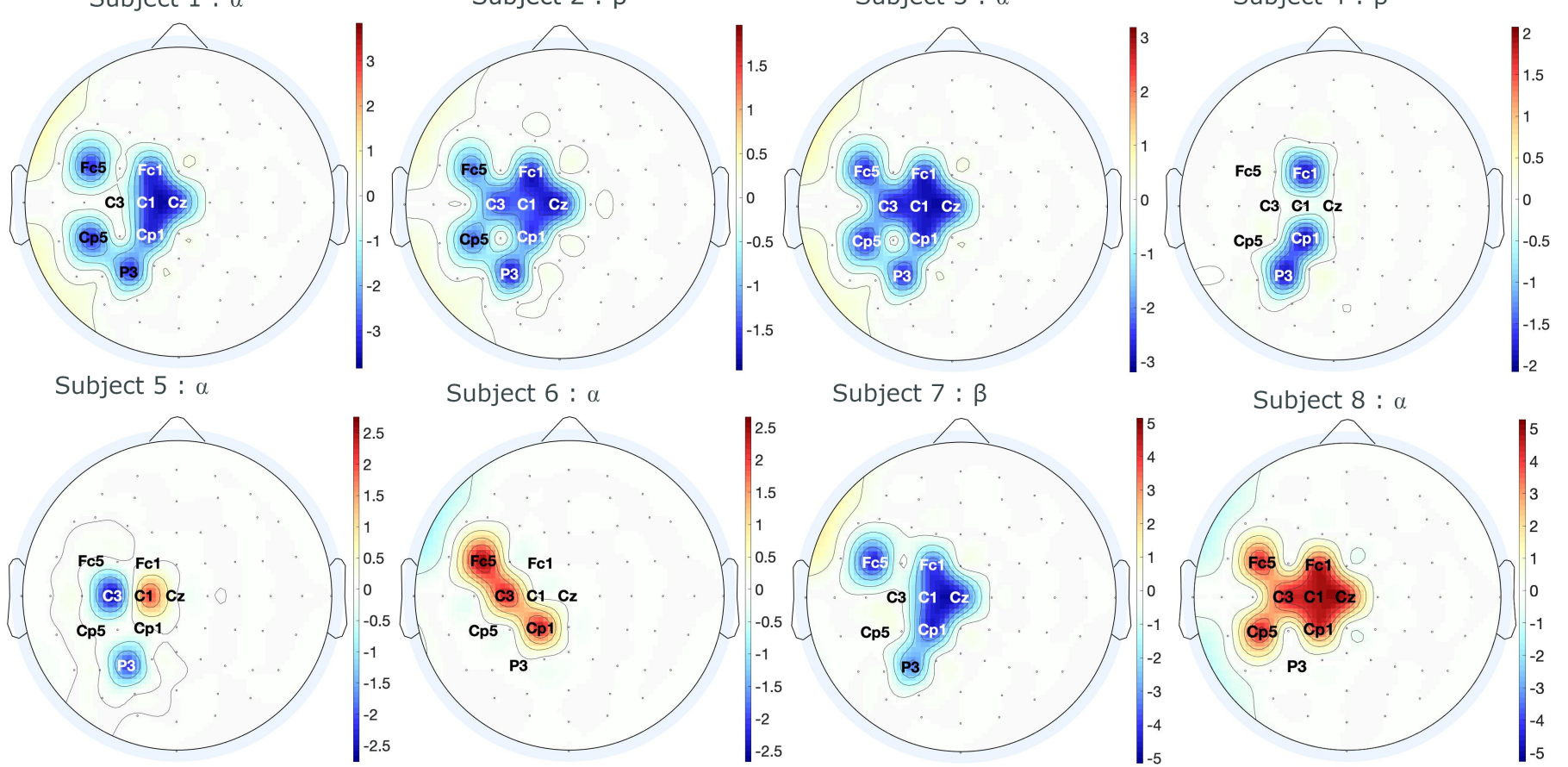

Subject $7: \beta$

Subject $8: \alpha$


Fig. 3. Subject-specific EEG power differences between Control by gaze and No Control. For each subject, the most significant frequency band ( $\alpha$ or $\beta$ ) in terms of T-value is illustrated $(\mathrm{p}<0.05$, FDR corrected

\begin{tabular}{|l|l|l|l|l|l|l|l|l|}
\hline Pair & 1 & 2 & 4 & 5 & 6 & 7 \\
\hline Mirror Vs Rest & $\begin{array}{l}\mathrm{t}=-5,34 \\
\mathrm{p}=0,001(\beta)\end{array}$ & $\begin{array}{l}\mathrm{t}=-1.80 \\
\mathrm{p}=0.001(\alpha)\end{array}$ & $\begin{array}{l}\mathrm{t}=-2.51 \\
\mathrm{p}=0.015(\alpha)\end{array}$ & $\begin{array}{l}\mathrm{t}=1.15 \\
\mathrm{p}=0.015(\beta)\end{array}$ & $\begin{array}{l}\mathrm{t}=-8.91 \\
\mathrm{p}=0.001(\beta)\end{array}$ & $\begin{array}{l}\mathrm{t}=1.98 \\
\mathrm{p}=0.019(\beta)\end{array}$ & $\begin{array}{l}\mathrm{t}=-8.29 \\
\mathrm{p}=0.001(\alpha)\end{array}$ & $\begin{array}{l}\mathrm{t}=-2.69 \\
\mathrm{p}=0.015(\alpha)\end{array}$ \\
\hline Gaze Vs Rest & $\mathrm{NS}$ & $\begin{array}{l}\mathrm{t}=-1.81 \\
\mathrm{p}=0.017(\alpha)\end{array}$ & $\mathrm{NS}$ & $\mathrm{NS}$ & $\begin{array}{l}\mathrm{t}=-1.00 \\
\mathrm{p}=0.012(\beta)\end{array}$ & $\begin{array}{l}\mathrm{t}=-2.17 \\
\mathrm{p}=0.045(\alpha)\end{array}$ & $\begin{array}{l}\mathrm{t}=-1.13 \\
\mathrm{p}=0.019(\beta)\end{array}$ & $\begin{array}{l}\mathrm{t}=-4.66 \\
\mathrm{p}=0.001(\alpha)\end{array}$ \\
\hline No Control Vs Rest & $\begin{array}{l}\mathrm{t}=3.73 \\
\mathrm{p}=0.010(\beta)\end{array}$ & $\begin{array}{l}\mathrm{t}=-1.72 \\
\mathrm{p}=0.010(\alpha)\end{array}$ & $\mathrm{NS}$ & $\mathrm{NS}$ & $\begin{array}{l}\mathrm{t}=-3.1943 \\
\mathrm{p}=0.002(\alpha)\end{array}$ & $\begin{array}{l}\mathrm{t}=-1.78 \\
\mathrm{p}=0.006(\beta)\end{array}$ & $\mathrm{NS}$ & $\begin{array}{l}\mathrm{t}=-3.98 \\
\mathrm{p}=0.001(\alpha)\end{array}$ \\
\hline
\end{tabular}

TABLE I

SUBJECT-SPECIFIC EEG POWER DIFFERENCES BETWEEN CONTROL CONDITIONS AND REST. FOR EACH SUBJECT, THE MOST SIGNIFICANT FREQUENCY BAND $(\alpha$ OR $\beta$ ) IN TERMS OF T-VALUE IS ILLUSTRATED $(\mathrm{P}<0.05$, FDR CORRECTED $)$; NS=NON SIGNIFICANT $)$

[6] K. Kilteni et al. "The Sense of Embodiment in Virtual Reality". In: Presence: Teleoperators and Virtual Environments 21.4 (2012), pp. 373-387.

[7] JP. Van Acken. "Tracking the Sense of Agency in BCI Applications". PhD thesis. 2012.

[8] Q.B. Pham et al. "Skeleton Formation From Human Silhouette Images Using Joint Points Estimation". In: Second International Conference on Advances in Computing, Control and Communication Technology. 2018, pp. 101-105.

[9] S. R. Buss. "Introduction to Inverse Kinematics with Jacobian Transpose, Pseudoinverse and Damped Least Squares methods". In: IEEE Transactions on Robotics and Automation (2004), p. 19.

[10] A. Delorme and S. Makeig. "EEGLAB: an open source toolbox for analysis of single-trial EEG dynamics including independent component analysis". In: Journal of Neuroscience Methods 134.1 (2004), pp. 9-21.
[11] F. Tadel et al. "Brainstorm: A User-Friendly Application for MEG/EEG Analysis". In: Computational Intelligence and Neuroscience (2011), pp. 1-13.

[12] T. Mulder. "Motor imagery and action observation: cognitive tools for rehabilitation". In: Journal of Neural Transmission 114.10 (2007), pp. 1265-1278.

[13] W. Klimesch. "Alpha-band oscillations, attention, and controlled access to stored information". In: Trends in Cognitive Sciences 16.12 (2012), pp. 606-617.

[14] R. J. Gentili and C. Papaxanthis. "Laterality effects in motor learning by mental practice in right-handers". In: Neuroscience 297 (2015), pp. 231-242. 